\title{
Neonatal capsaicin treatment in rats induces chronic hyperthermia resulting in infectious disease
}

\author{
KEUN-YEONG JEONG and HWAN MOOK KIM
}

College of Pharmacy, Gachon Institute of Pharmaceutical Sciences, Gachon University, Incheon 406-840, Republic of Korea

Received January 13, 2015; Accepted September 1, 2015

DOI: $10.3892 /$ etm.2015.2829

\begin{abstract}
Treatment of neonatal animals with capsaicin has previously been associated with long-lasting hyperthermia and severe cutaneous lesions. The present study analyzed the effects of capsaicin-induced hyperthermia on the occurrence of infectious disease and pruritic dermatitis in a rat model. Pregnant Sprague-Dawley (SD) rats were obtained 1 week prior to parturition. Pups from each litter were randomly assigned to the following experimental groups: Capsaicin-treated (cap-treated; $n=10)$ or vehicle-treated $(n=5)$. Capsaicin $(50 \mathrm{mg} / \mathrm{kg}$ ) or vehicle were systemically administered to the SD rat pups (age, $48 \mathrm{~h}$ ), after which body temperature was measured using a biotelemetry system, and the effects of hyperthermia on the ability of the rat pups to resist bacterial infection were analyzed. Furthermore, pruritus-induced scratching behavior and dermatitis were assessed, and changes in interleukin (IL)-4- and IL-13-induced immunoglobulin E expression were measured. Treatment of neonatal rats with capsaicin resulted in chronic hyperthermia, which had negative effects on the host immune defense response. The expression levels of T-helper type 2 cell-associated cytokines were significantly increased $(\mathrm{P}<0.01)$ in the cap-treated rats following bacterial infection with Staphylococcus aureus or Streptococcus agalactiae. Furthermore, cap-treated rats exhibited pruritus-induced scratching behavior and dermatitis. The results of the present study suggested that treatment of neonatal rats with capsaicin induces chronic hyperthermia and decreases the effectiveness of the host defense system. Therefore, a cap-treated neonatal rat model may be considered useful when investigating the association between hyperthermia and infectious disease.
\end{abstract}

Correspondence to: Professor Keun-Yeong Jeong or Professor Hwan Mook Kim, College of Pharmacy, Gachon Institute of Pharmaceutical Sciences, Gachon University, Hambangmoe-ro 191, Incheon 406-840, Republic of Korea

E-mail: alvirus@naver.com

E-mail: hwanmook@gachon.ac.kr

Abbreviations: BAT, brown adipose tissue; cap-treated, capsaicin-treated; IL, interleukin; Ig, immunoglobulin; TRPV1, transient receptor potential vanilloid 1 ; RBD3, rat $\beta$-defensin 3

Key words: neonatal capsaicin treatment, rat, hyperthermia, bacterial infection, pruritus

\section{Introduction}

The metabolic function of brown adipose tissue (BAT) contributes to the maintenance of body temperature during cold exposure, and to the elevated core temperature during various behavioral states, including the acute phase response and stress (1). BAT contains numerous cell types in addition to adipocytes, including pericytes, monocytes and macrophages; therefore, it has a critical role in the immune response (2). Numerous studies have investigated the thermal regulation and immunological functions of BAT $(3,4)$.

The transient receptor potential vanilloid 1 (TRPV1) channel, which is a member of a large family of transient receptor potential ion channels (5), is a ligand-gated, non-selective cation channel that is permeable to $\mathrm{Ca}^{2+}$. Numerous studies have investigated the function of TRPV1 and have proposed various sensory mechanisms. Tékus et al (6) demonstrated that blocking TRPV1 with various antagonists resulted in acute hyperthermia in rodents; thus suggesting that TRPV1 may be involved in regulating body temperature in vivo (9). However, this effect was not observed for TRPV1-knockout mice $(7,8)$. TRPV1 is activated by noxious heat, protons and various endogenous factors in vitro (10), and capsaicin and capsazepine have previously been demonstrated to be specific ligands of TRPV1 (11). Capsaicin activates TRPV1, whereas capsazepine inhibits TRPV1 $(11,12)$.

Capsaicin is the predominant constituent of hot chilli peppers, and is responsible for their spicy and strong flavor. In a previous study, treatment of neonatal rats with capsaicin was associated with neurotoxic effects, including the destruction of a subset of small-diameter primary afferents (13); thus suggesting that capsaicin may be a useful tool for investigating TRPV1-mediated sensory fiber functions, including taste, pain and thermosensation $(14,15)$. Hypersensitivity associated with immunoglobulin ( $\mathrm{Ig}) \mathrm{E}$ mediates pathological pruritus; however, the exact etiology remains unknown. The pathogenesis of hypersensitivity involves a complex immunologic cascade, including disruption of the epidermal barrier. The major elements in immune dysregulation are Langerhans' cells, inflammatory dendritic epidermal cells and mast cells, all of which interact through an intricate cascade of cytokines leading to a predominance of $\mathrm{Th}_{2}$ cells. $\mathrm{The}^{\mathrm{T}} \mathrm{Th}_{2}$ cytokines: Interleukin (IL)-4, IL-5, IL-10 and IL-13, are therefore increased in the skin (16). Leptin is an adipocyte-derived hormone. Recently, leptin has been shown to modulate innate 
immune responses such as cytokine synthesis, in vitro, and has been shown to have a role in the innate host response against bacteria in vivo (17)

In our previous study, we investigated the effects of capsaicin on neonatal Sprague-Dawley rat pups, and consistently demonstrated long-lasting hyperthermia and severe cutaneous lesions on their heads, necks and backs, associated with vigorous scratching behavior. The present study evaluated the effects of capsaicin-induced hyperthermia on the immune function of rat neonates, including their ability to resist bacterial infections.

\section{Materials and methods}

Rats. The rat facilities were approved by the Association of Assessment and Accreditation of Laboratory Animal Care, and animal experiments were performed according to the institutional guidelines outlined by the Institutional Animal Care and Use Committee at Gachon University (LCDI-2014-0082; Incheon, Republic of Korea). Pregnant Sprague-Dawley rats (Samtako, Seong-nam, Republic of Korea) were obtained 1 week prior to parturition, housed individually in plastic cages with soft bedding, and allowed to deliver. Pups from each litter were randomly assigned to an experimental group, weaned 21 days postnatally, separated on the basis of gender, and housed in groups of 3-5 pups until the end of the experiment. Only the male pups were used in the present study, including 10 in the capsaicin-treated (cap-treated) group and 5 in the vehicle-treated group. All female rats were sacrificed by $\mathrm{CO}_{2}$ inhalation. All of the rats were maintained in a $12 \mathrm{~h}$ light/dark cycle (light on, 8:00 AM) at $22-25^{\circ} \mathrm{C}$, with free access to food and water.

TRPV1 antagonist. Capsazepine (Sigma-Aldrich, St. Louis, MO, USA) was dissolved in phosphate-buffered saline (PBS), and $50 \mathrm{mg} / \mathrm{kg}$ capsazepine was injected intraperitoneally into 6 -week-old rats. Untreated 6-week-old naïve rats were used as untreated controls.

Neonatal capsaicin treatment to induce hyperthermia. Capsaicin (Sigma-Aldrich) was suspended in PBS containing 10\% Tween 80 (Sigma-Aldrich) and 10\% ethanol, using the method outlined in Kim et al (18). Subsequently, capsaicin $(50 \mathrm{mg} / \mathrm{kg}$, cap-treated) or an equal volume of saline containing 10\% Tween 80 and 10\% ethanol (vehicle-treated), were systemically administered to SD rat pups within $48 \mathrm{~h}$ of birth.

Measurement of body temperature. The body temperatures of rat pups were measured using small implantable transponders (PDT-4000; Mini-Mitter, Co., Inc., Bend, OR, USA) that were implanted into the abdominal cavity of the rats, following anesthetization using isoflurane (0.5-2\%; Hana Pharm. Co., Ltd, Seoul, South Korea). Temperature data were constantly received using an ER4000 receiver (56x29x7 cm; RS 232 serial; Mini-Mitter, Co., Inc.), and automatically recorded onto a main computer using PDT-4000 software (Mini-Mitter, Co., Inc.).

Reverse transcription-quantitative polymerase chain reaction (RT-qPCR). Rat L5 dorsal root ganglion (DRG) and skin samples were obtained following sacrifice of the rat pups with $\mathrm{CO}_{2}$ inhalation. Total RNA from each tissue was extracted using an RNeasy ${ }^{\circledR}$ Micro kit (Qiagen, Venlo, Limburg, Netherlands), according to the manufacturer's instructions. Subsequently, total RNA was reverse transcribed to cDNA using a reverse transcription system (Promega Corporation, Madison, WI, USA). qPCR was performed for the rat L5 DRG total RNA sample, using a total reaction volume of $20 \mu \mathrm{l}$ containing $10 \mu \mathrm{l} \mathrm{SYBR}{ }^{\circledR}$ Green PCR Master mix (Applied Biosystems, Grand Island, NY, USA), a primer pair (1 $\mu$ l each of $10 \mathrm{pmol} / \mu \mathrm{l}$ primers), and $8 \mu \mathrm{l}$ diluted cDNA (500 $\mathrm{ng} / \mu \mathrm{l})$. qPCR was performed for the skin total RNA sample using PCR pre-mixture (Bioneer, Seong-Nam, Korea), a primer pair ( $1 \mu \mathrm{l}$ each of $10 \mathrm{pmol} / \mu \mathrm{l}$ primers) and $8 \mu \mathrm{l}$ diluted cDNA (500 ng/ $/ \mathrm{l}$ ). The PCR cycling conditions were as follows: $95^{\circ} \mathrm{C}$ for $10 \mathrm{sec}, 55^{\circ} \mathrm{C}$ for $20 \mathrm{sec}$ and $72^{\circ} \mathrm{C}$ for $30 \mathrm{sec}$, for 40 cycles and the initial denaturation and final extension conditions were $95^{\circ} \mathrm{C}$ (5 mins) and $72^{\circ} \mathrm{C}$ (10 mins). Relative expression levels were determined in comparison with the GAPDH gene, or using the $2^{-\Delta \Delta \mathrm{Ct}}$ method (19). The primer pairs for rat TRPV1, IL-4, IL-13 and GAPDH are listed in Table I.

ELISA. Blood samples were collected from rats following gas anesthetization using isoflurane $(0.5 \%-2 \%)$. The samples were centrifuged at 7,500 x g for $30 \mathrm{~min}$ and the supernatants, corresponding to the blood serum, were collected. Total protein concentrations for each serum sample were determined using a bicinchoninic acid (BCA) assay (Pierce Biotechnology, Inc., Rockford, IL, USA). Protein expression levels of leptin, IL-4, IL-13, and IgE, were measured using a Rat ELISA Quantitation kit (Bethyl Laboratories, Inc., Montgomery, TX, USA), according to the manufacturer's instructions.

Western blot. Skin samples were collected from cap- and vehicle-treated rats, following anesthesia with intraperitoneally injected pentobarbital (50 mg/kg; Sigma-Aldrich). The skin samples $(2 \mu \mathrm{g})$ were homogenized in T-per tissue lysis buffer (20 $\mu$ l; Thermo Fisher Scientific, Inc., Waltham, MA, USA) containing protease inhibitors (Thermo Fisher Scientific, Inc.), the homogenates were centrifuged at $10,000 \mathrm{x} g$ for $5 \mathrm{~min}$ and the protein supernatant was collected. Total protein concentrations for each sample were determined using a BCA assay (Pierce Biotechnology, Inc.). Protein extracts $(30 \mathrm{mg})$ were separated by $12 \%$ sodium dodecyl sulfate-polyacrylamide gel electrophoresis at $100 \mathrm{~V}$ and $25 \mathrm{~mA}$ for $2 \mathrm{~h}$ (Bio-Rad Laboratories, Inc., Hercules, CA, USA). The separated proteins were transferred onto a protran-nitrocellulose membrane (GE Healthcare Bio-Sciences, Pittsburgh, PA, USA), blocked for $1 \mathrm{~h}$ in blocking buffer (5\% non-fat powdered milk in Tris-buffered saline containing Tween 20), and then incubated for $24 \mathrm{~h}$ in 1:500 diluted rabbit anti-rat $\beta$-defensin 3 (RBD3) polyclonal antibody (1:500; cat. no. NB200-117; Novus Biologicals, LLC, Littleton, CO, USA) and rabbit anti-rat GAPDH polyclonal antibodies (1:5,000; cat. no. NB100-56875; Novus Biologicals, LLC). The membranes were incubated in 1:1,000 diluted horseradish peroxidase-conjugated goat anti-rabbit IgG (bs-0295G-HRP) Bioss Antibodies, Woburn, MA, USA) for $1 \mathrm{~h}$ at room temperature. Antibody complexes were detected using a chemiluminescent peroxidase substrate (Sigma-Aldrich), and developed using X-ray film and developer (Agfa, Mortsel, 
Table I. Primer pairs for polymerase chain reaction.

\begin{tabular}{lcc}
\hline Gene & \multicolumn{1}{c}{ Forward } & Reverse \\
\hline TRPV1 & GGCTCCGGTACTTCTCTTTC & AATAGGGGAGTGGTCAAAGG \\
IL-4 & CATGGCCAGTGTGCAGAGAG & GAGGCCACCAAACAGACAGG \\
IL-13 & AACCCGTGGACCAAGGAAGT & GTGAGCTGTGGGAAGGTTGG \\
GAPDH & AACCCGTGGACCAAGGAAGT & GTGAGCTGTGGGAAGGTTGG
\end{tabular}

TRPV1, transient receptor potential vanilloid 1; IL, interleukin.

A

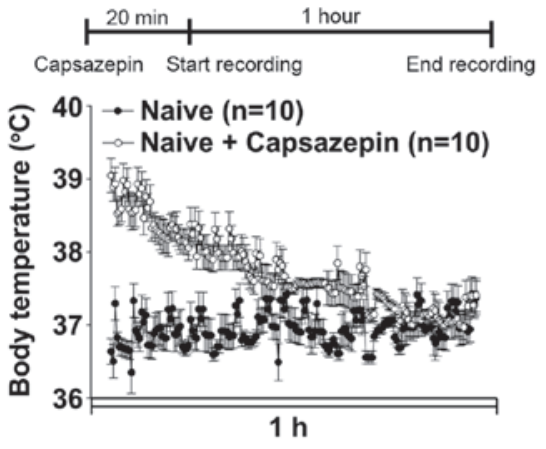

C

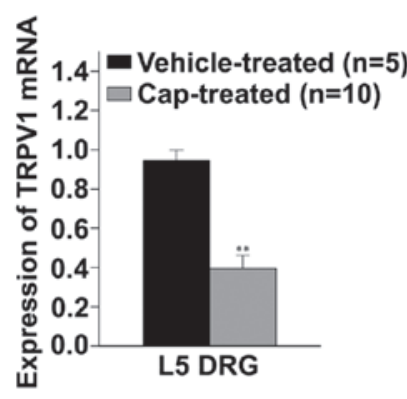

D

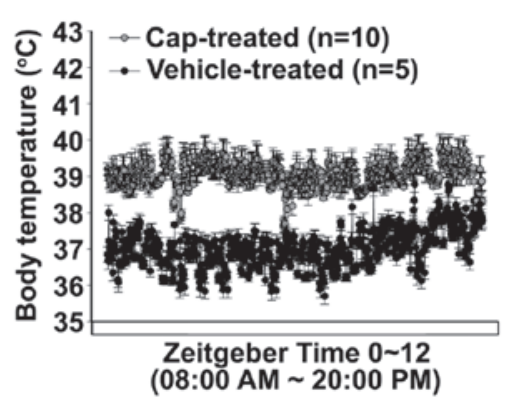

B

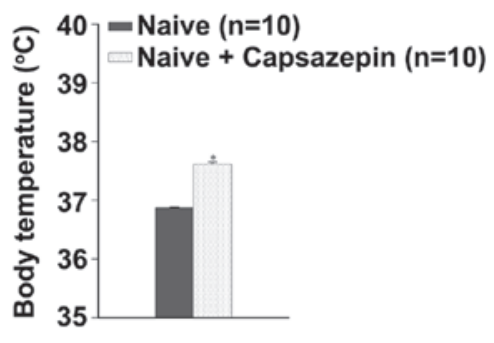

$\mathbf{E}$

Figure 1. Inducement of hyperthermia via the transient receptor potential vanilloid 1 (TRPV1) channel. (A) Measurement of body temperature following intraperitoneal injection of capsazepine $(50 \mathrm{mg} / \mathrm{kg}$ ) into rats. (B) Quantitative analysis of changing body temperature. (C) Quantitative analysis of TRPV1 mRNA expression levels in the L5 dorsal root ganglion of vehicle-treated and capsaicin (cap)-treated rats. (D) Measurement of body temperature in the vehicle- and cap-treated rats. (E) Quantitative analysis of changing body temperature. Data are mean \pm standard deviation. ${ }^{*} \mathrm{P}<0.05,{ }^{* *} \mathrm{P}<0.001 \mathrm{vs}$. control.

Belgium). Densitometry measurements were made using Image J software (National Institutes of Health, Bethesda, MD, USA).

Bacterial colonization. Skin samples from lesional or non-lesional epidermis were obtained from cap- or vehicle-treated rats via punching biopsies, following anesthetization using intraperitoneally administered pentobarbital (50 $\mathrm{mg} / \mathrm{kg}$ ). Bacterial colonies from the skin samples were grown on blood agar plates (Thermo Fisher Scientific, Inc.), after which the colonies were suspended in $100 \mu \mathrm{l}$ distilled water, inoculated onto Müller-Hinton agar plates and incubated at $37^{\circ} \mathrm{C}$ for $48 \mathrm{~h}$ at $5 \% \mathrm{CO}_{2}$. The number of colonies were counted as colony-forming units $/ \mathrm{cm}^{2}$. Bacterial identification was cross-checked using a conventional method (coagulase and mannitol fermentation tests) (20) and an automated identification system, VITEK $^{\circledR} 2$ (bioMérieux, Durham, NC, USA). Methicillin resistance was monitored using the Clinical and
Laboratory Standards Institute antimicrobial susceptibility method (21). Briefly, cefoxitin disks $(30 \mu \mathrm{g})$ were placed on Müller-Hinton agar, and an inhibition zone diameter of $\leq 21 \mathrm{~mm}$ was considered to indicate methicillin resistance.

Scratching behavior. Rats were placed into separate plastic chambers (room temperature; 200×300x200 mm; Daihan Bio, Seongnam, South Korea), equipped with a mirror behind the chamber, which allowed an unobstructed view. Following habituation, scratching behavior was recorded using an unmanned digital video camera (DCR-SR300; Sony, Tokyo, Japan). A bout of consecutive scratching strokes using the hind paw was regarded as one scratch.

Statistical analysis. All data are presented as the mean \pm standard deviation. Statistical significance was analyzed using the Student's t-test or the Mann-Whitney rank sum test, depending on normality. $\mathrm{P}<0.05$ was considered to indicate statistically 
A

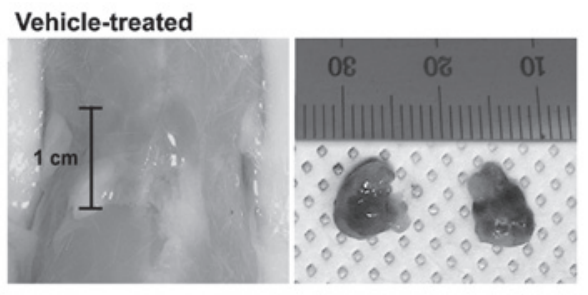

Cap-treated
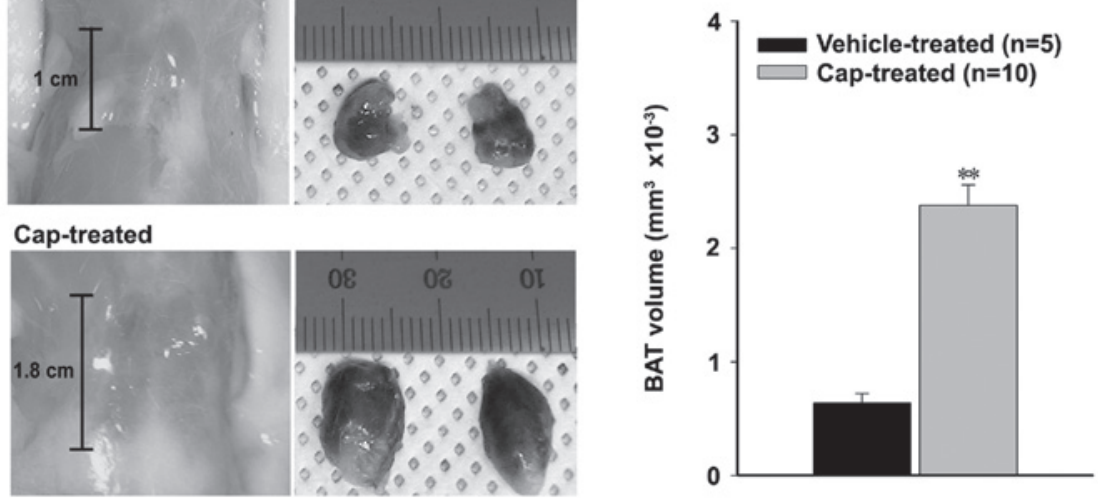

B

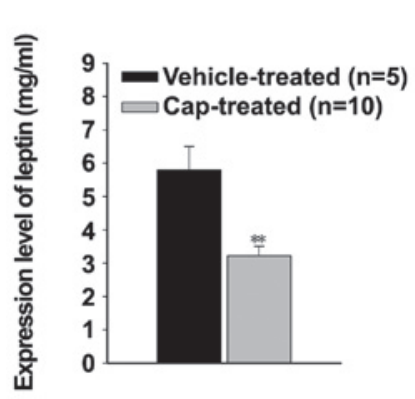

D
C
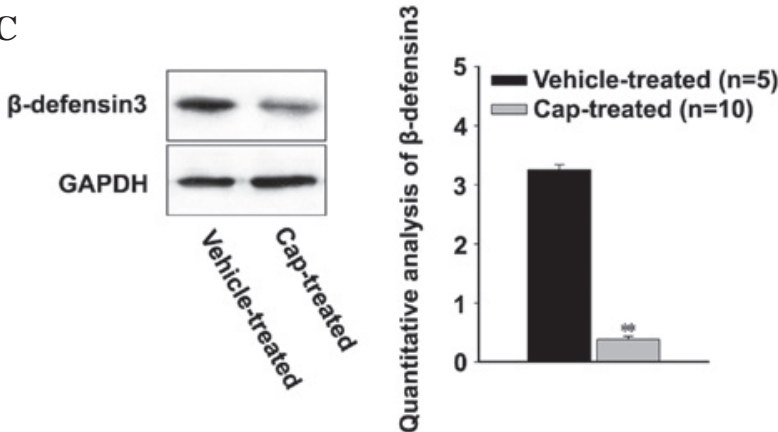

$\mathbf{E}$
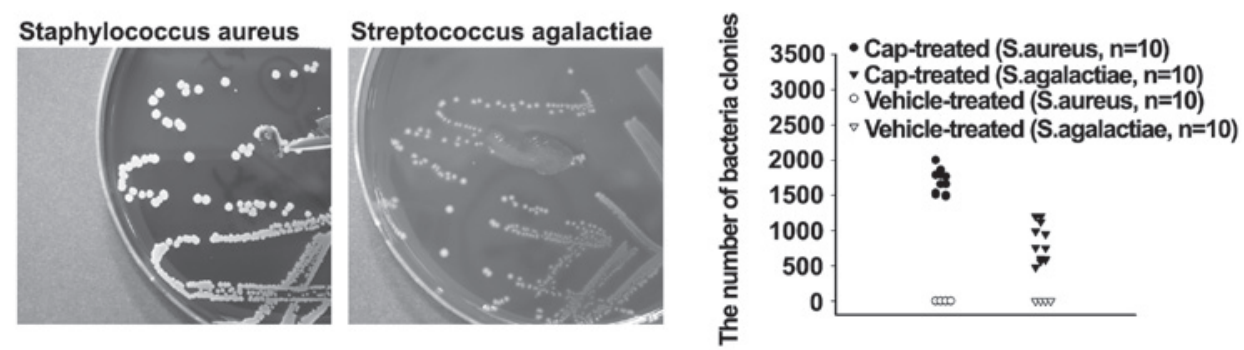

Figure 2. Decline in the host defense system. (A) A comparison of BAT size between the vehicle-treated and cap-treated rats. (B) Measurement of leptin expression levels. (C) Western blot analysis of rat $\beta$-defensin 3 (RBD3) expression levels (left). The right graph depicts the results of a quantitative analysis of RBD3 expression. (D) Staphylococcus aureus and Streptococcus agalactiae were grown on blood agar plates. (E) The number of bacterial colonies formed on the blood agar plates. Data are presented as the mean \pm standard deviation. ${ }^{*} \mathrm{P}<0.05,{ }^{* *} \mathrm{P}<0.001$ vs. control. BAT, brown adipose tissue; cap-treated, capsaicin-treated.

significant differences. All statistical analyses were conducted using SigmaStat software (version 3.5; Systat Software Inc., San Jose, IL, USA).

\section{Results}

Neonatal capsaicin treatment induces TRPVI knockdown-associated chronic hyperthermia in rats. Rat pups were treated with capsaicin (age, $48 \mathrm{~h}$ ) and capsazepine (age, 6 weeks), and alterations in body temperature were evaluated (Fig. 1). The capsazepine-treated rats demonstrated hyperthermic symptoms for $1 \mathrm{~h}$, and the core body temperature was markedly increased in these rats, as compared with the naïve rats $\left(37.61 \pm 0.03\right.$ and $36.8 \pm 0.01^{\circ} \mathrm{C}$, respectively; Fig. 1A and B). The expression levels of TRPV1 mRNA significantly decreased by $\sim 40 \%$ in the rat L5 DRG following neonatal capsaicin treatment, compared with the vehicle-treated rats $(\mathrm{P}<0.001$; Fig. 1C). Neonatal capsaicin treatment was associated with chronic hyperthermia; the body temperature significantly increased to $38.47 \pm 0.04^{\circ} \mathrm{C}$, compared with the vehicle-treated rats (body temperature, $36.86 \pm 0.01^{\circ} \mathrm{C}$; $\mathrm{P}<0.001$; Fig. 1D and E).

Chronic hyperthermia disrupts the immune defense against bacterial infection. In order to investigate the effects of hyperthermia on the immune systems of the rats, the sizes of interscapular BAT were compared. The mean length of BAT was $1 \mathrm{~cm}$ in the vehicle-treated rats and $1.8 \mathrm{~cm}$ in the cap-treated rats (Fig. 2A, left). Furthermore, the mean volume of BAT significantly increased in the cap-treated rats, compared with the vehicle-treated rats $(\mathrm{P}<0.001 ;$ Fig. $2 \mathrm{~A}$, right). Conversely, the expression levels of leptin were significantly decreased in the cap-treated rats, compared with the vehicle-treated rats $(\mathrm{P}<0.001$; Fig. $2 \mathrm{~B})$. The expression levels of RBD3 were 


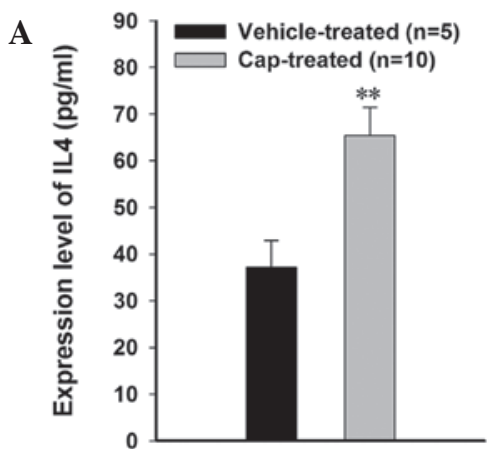

C

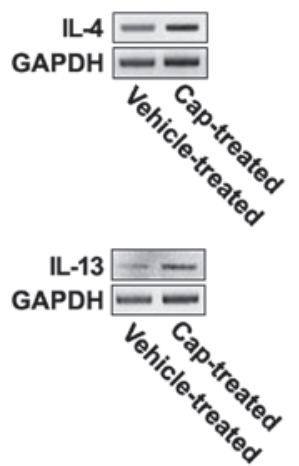

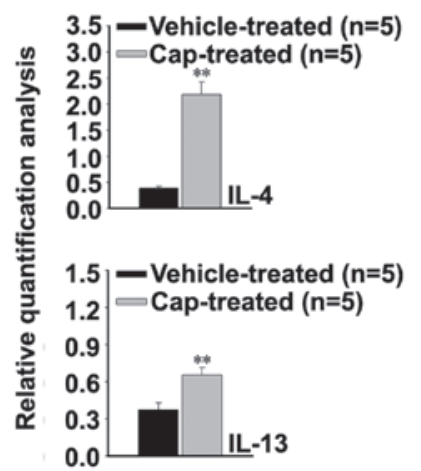

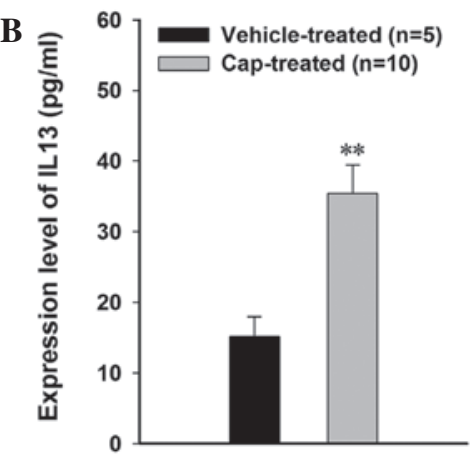

D

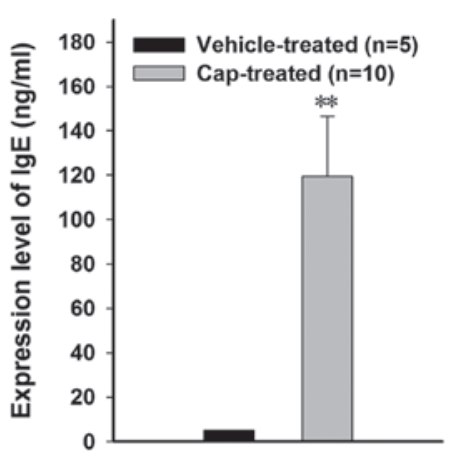

Figure 3. Pruritus-associated T-helper type 2 cell cytokine expression levels. The measurement of (A) IL-4 and (B) IL-13 protein expression levels in the serum of vehicle-treated and cap-treated rats. (C) Endogenous expression levels of IL-4 and IL-13 genes in the skin of vehicle-treated and cap-treated rats. (D) The measurement of IgE expression levels in the serum of vehicle-treated and cap-treated rats. Data are presented as the mean \pm standard deviation. ${ }^{* *} \mathrm{P}<0.001 \mathrm{vs}$. control. IL, interleukin; cap-treated, capsaicin-treated; Ig, immunoglobulin.

A

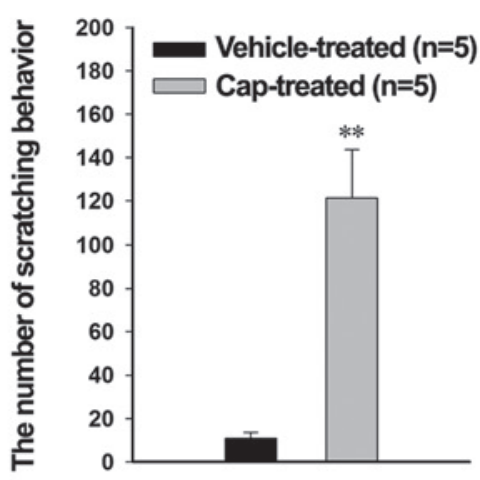

B

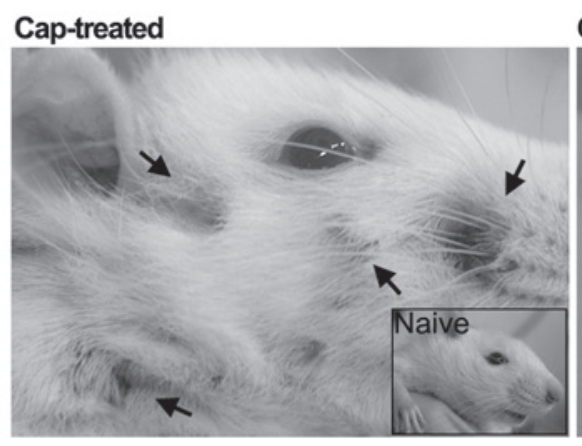

Cap-treated

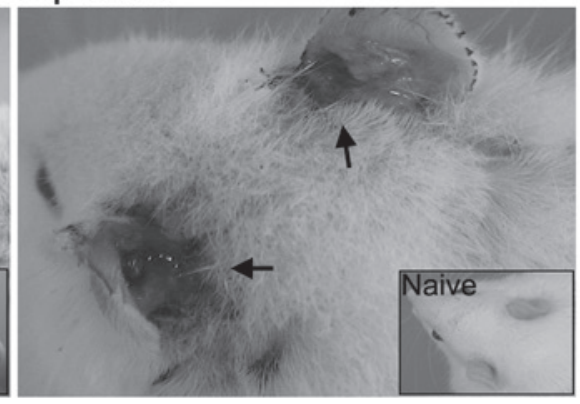

Figure 4. Observation of scratching behavior and dermatitis. (A) Measurement of scratching behavior in cap-treated and vehicle-treated rats. Data are presented as the mean \pm standard deviation. ${ }^{* *} \mathrm{P}<0.001$ vs. control. (B) Severe scratches and dermatitis were detected in cap-treated rats. Cap-treated, capsaicin-treated.

investigated in order to understand the effects of decreased levels of leptin on the host defense system. According to the western blot, expression levels of RBD3 were significantly decreased in the cap-treated rats $(\mathrm{P}<0.001$; Fig. 2C). Bacterial infection was confirmed by growth on blood agar plates, and the number of colonies were ascertained using conventional and automated colony counting assays (Fig. 2D and E). Up to 2,000 colonies of Staphylococcus aureus and 1,200 colonies of Streptococcus agalactiae were identified in the cap-treated rats. Conversely, no bacterial infection could be identified in the vehicle-treated rats (Fig. 2E).
Bacterial infection induces dysregulation of pruritus-associated cytokines. In order to investigate the effects of bacterial infection on the levels of $\mathrm{Th}_{2}$-associated cytokines, the blood serum protein expression levels of IL-4 and IL-13 were measured, and were demonstrated to have significantly increased in the cap-treated rats following bacterial infection, as compared with the vehicle-treated rats $(\mathrm{P}<0.001$; Fig. 3A and B). The endogenous expression levels of IL-4 and IL-13 mRNA were investigated in lesional and non-lesional skin samples from the rats, and both cytokines were significantly increased in the cap-treated rats, compared 
with the vehicle-treated rats $(\mathrm{P}<0.001 ; \mathrm{Fig} .3 \mathrm{C})$. In addition, upregulation of the $\mathrm{Th}_{2}$-associated cytokines was associated with significantly increased expression levels of $\operatorname{IgE}$ in the cap-treated rats $(\mathrm{P}<0.001$; Fig. 3D).

Increased expression levels of pruritic-associated cytokines evoke scratching behavior and dermatitis. In order to investigate the effects of the pruritic-associated cytokines, the scratching behavior of the rats was observed using a digital video camera. Pruritus-induced scratching behavior was significantly increased in the cap-treated rats after $1 \mathrm{~h}$, compared with the vehicle-treated rats $(121.29 \pm 22.48$ and $10.81 \pm 2.76$ times, respectively; $\mathrm{P}<0.001$; Fig. $4 \mathrm{~A}$ ). Concordantly, nail marks and signs of inflammatory bleeding were detected on the face, behind the ears, and on the nape of the neck of the cap-treated rats, whereas the vehicle-treated rats exhibited a normal appearance (Fig. 4B); these regions are easily accessible to the rat hind paws.

\section{Discussion}

In our previous study, treatment of rats with capsaicin was associated with long-lasting hyperthermia and severe cutaneous lesions; therefore, the present study aimed to investigate the effects of capsaicin-induced hyperthermia on the susceptibility of neonatal rats to bacterial infections, in particular pruritic dermatitis.

In the present study, treatment with capsazepine markedly increased the core body temperature of naïve rats, in line with a previous study in which blocking TRPV1 was associated with an increased body temperature (9); thus suggesting that TRPV1 may have a role in thermoregulation. The present study demonstrated that the use of capsazepine in the generation of a rat hyperthermia model is limited, as it is only able to increase the core body temperature of rats for a short period of time, due to its limited duration of activity. By contrast, treatment of neonatal rats with capsaicin initiated long-lasting hyperthermia, and reduced the expression of TRPV, in a manner that mimicked the effects of TRPV1 antagonists (22).

BAT is present and active in human newborns, in which it is responsible for maintaining body temperature, and is essential for classical non-shivering thermogenesis (1); therefore, BAT activity may be affected by an abnormal increase in core body temperature. It is generally accepted that BAT is rapidly lost postnatally, and that this process is normally concluded within the first few years of life (23); however, in the present study, the size of BAT increased in cap-treated adult rats. This abnormal increase in the size of BAT may have been indicative of problems with thermogenic regulation.

BAT thermogenesis is important for the maintenance of normothermia when small animals are exposed to a cold environment (24). Therefore, we hypothesized that the BAT of cap-treated rats may be affected by a thermogenic regulation disorder, such as chronic hyperthermia. As the regulation of in vivo metabolism is an additional function of the BAT, the body weight of the rats was expected to alter in response to hyperthermia (25); however, there was no significant difference in body weight between the vehicle-treated and cap-treated rats (data not shown).
Leptin is synthesized exclusively by adipocytes and acts to regulate the balance of energy. Previous studies investigating mRNA expression levels of leptin demonstrated that leptin is expressed in the skeletal muscle, particularly in BAT (26). The most important biological activities attributed to leptin include effects on feeding, metabolism and the neuroendocrine axis (27). Numerous studies have detected elevated serum expression levels of leptin in humans and mice during the early phase of sepsis, following systemic endotoxin administration, and during the acute phase response (28). Furthermore, a deficiency in leptin has been associated with an increased frequency of infection (29); therefore, in the present study, decreased leptin expression levels may have increased the susceptibility of the rats to bacterial infections.

Leptin contributes to cutaneous antimicrobial defense systems by upregulating the expression of defensins, although the underlying mechanism of this is yet to be elucidated $(30,31)$. Defensins are a family of antimicrobial peptides secreted by epidermal keratinocytes, in particular in response to cutaneous infections or in inflammatory diseases (32). Defensins have been demonstrated to contribute to the innate host defense via direct bacteriocidal activity (31). In particular, BD3 exhibits antibacterial activity towards gram-positive bacteria under physiological salt concentrations, and has significant involvement in adaptive immunity, compared with other defensins (33); thus suggesting that the leptin-associated decreased expression levels of BD3 in the cap-treated rats may have initiated immune dysfunction of the skin barrier, leading to a decline in the host defense and enhanced susceptibility to bacterial infections, including S. aureus and S. agalactiae.

It has been reported that the acute skin lesions of pruritus patients contain increased numbers of cells expressing IL-4 and IL-13 mRNA. IL-4 and IL-13 are pleiotropic cytokines that have a central role in IgE-dependent inflammatory reactions (34). IL-4 has an important role in stimulating B cells to produce IgE antibodies, and in the differentiation of Th cells into the $\mathrm{Th}_{2}$ phenotype. IL-13 similarly induces B cells to produce IgE, and IL-4 and IL-13 operate through the IL-4R and IL-13R receptors, respectively (34). In the present study, hyperthermia-induced bacterial infections in the cap-treated rats were associated with elevated expression levels of the $\mathrm{Th}_{2}$ cytokines, IL-4 and IL-13, which may have resulted in the occurrence of pruritic dermatitis (35).

In conclusion, the results of the present study suggest that treatment of neonatal rats with capsaicin induces chronic hyperthermia, which may have increased the susceptibility of the rats to bacterial infections. Bacterial infections in turn were associated with upregulated expression of the $\mathrm{Th}_{2}$-associated cytokines, which may have resulted in pruritus-induced scratching behavior and dermatitis in the cap-treated rats. Therefore, a capsaicin-induced chronic hyperthermia rat model may be useful for investigating the association between hyperthermia and infectious disease $(36,37)$.

\section{Acknowledgements}

The present study was supported by the Gachon Institute of Pharmaceutical Sciences Research Fund 2014, Gachon, Gachon University, Seongnam, South Korea. 


\section{References}

1. Morrison SF, Madden CJ and Tupone D: Central neural regulation of brown adipose tissue thermogenesis and energy expenditure. Cell Metab 19: 741-756, 2014.

2. Desruisseaux MS, Nagajyothi, Trujillo ME, Tanowitz HB and Scherer PE: Adipocyte, adipose tissue, and infectious disease. Infect Immun 75: 1066-1078, 2007.

3. Lateef DM, Abreu-Vieira G, Xiao C and Reitman ML: Regulation of body temperature and brown adipose tissue thermogenesis by bombesin receptor subtype-3. Am J Physiol Endocrinol Metab 306: E681-E687, 2014.

4. Wen JJ, Nagajyothi F, Machado FS, Weiss LM, Scherer PE, Tanowitz HB and Garg NJ: Markers of oxidative stress in adipose tissue during Trypanosoma cruzi infection. Parasitol Res 113: 3159-3165, 2014

5. Szallasi A, Cortright DN, Blum CA and Eid SR: The vanilloid receptor TRPV1: 10 years from channel cloning to antagonist proof-of-concept. Nat Rev Drug Discov 6: 357-372, 2007.

6. Tékus V, Bölcskei K, Kis-Varga A, Dézsi L, Szentirmay E, Visegrády A, Horváth C, Szolcsányi J and Petho G: Effect of transient receptor potential vanilloid 1 (TRPV1) receptor antagonist compounds SB705498, BCTC and AMG9810 in rat models of thermal hyperalgesia measured with an increasing-temperature water bath. Eur J Pharmacol 641: $135-141,2010$.

7. Gavva NR, Bannon AW, Hovland DN Jr, Lehto SG, Klionsky L, Surapaneni S, Immke DC, Henley C, Arik L, Bak A, et al: Repeated administration of vanilloid receptor TRPV1 antagonists attenuates hyperthermia elicited by TRPV1 blockade. J Pharmacol Exp Ther 323: 128-137, 2007.

8. Szelényi Z, Hummel Z, Szolcsányi J and Davis JB: Daily body temperature rhythm and heat tolerance in TRPV1 knockout and capsaicin pretreated mice. Eur J Neurosci 19: 1421-1424, 2004

9. Gavva NR, Bannon AW, Surapaneni S, Hovland DN Jr, Lehto SG, Gore A, Juan T, Deng H, Han B, Klionsky L: The vanilloid receptor TRPV1 is tonically activated in vivo and involved in body temperature regulation. J Neurosci 27: 3366-3374, 2007.

10. Caterina MJ, Schumacher MA, Tominaga M, Rosen TA, Levine JD and Julius D: The capsaicin receptor: A heat-activated ion channel in the pain pathway. Nature 389: 816-824, 1997.

11. Lambert DG: Capsaicin receptor antagonists: A promising new addition to the pain clinic. Br J Anaesth 102: 153-155, 2009.

12. Valenzano KJ and Sun Q: Current perspectives on the therapeutic utility of VR1 antagonists. Curr Med Chem 11: 3185-3202, 2004.

13. Hiura A: Neuroanatomical effects of capsaicin on the primary afferent neurons. Arch Histol Cytol 63: 199-215, 2000.

14. Benham CD, Davis JB and Randall AD: Vanilloid and TRP channels: A family of lipid-gated cation channels. Neuropharmacology 42: 873-888, 2002.

15. Tominaga M: Function of TRP channel as a thermal receptor Nihon Seirigaku Zasshi 65: 130-137, 2003. (In Japanese).

16. Leung DY and Bieber T: Atopic dermatitis. Lancet 361: 151-160, 2003.

17. Mancuso P, Gottschalk A, Phare SM, Peters-Golden M, Lukacs NW and Huffnagle GB: Leptin-deficient mice exhibit impaired host defense in Gram-negative pneumonia. J Immunol 168: 4018-4024, 2002.

18. Kim YI, Na HS, Han JS and Hong SK: Critical role of the capsaicin-sensitive nerve fibers in the development of the causalgic symptoms produced by transecting some but not all of the nerves innervating the rat tail. J Neurosci 15: 4133-4139, 1995.

19. Livak KJ and Schmittgen TD: Analysis of relative gene expression data using real-time quantitative PCR and the 2(-Delta Delta C(T)) Method. Methods 25: 402-408, 2001.
20. Raymond EA and Traub WH: Identification of staphylococci isolated from clinical material. Appl Microbiol 19: 919-922, 1970.

21. Cuenca-Estrella M, Gomez-Lopez A, Alastruey-Izquierdo A, Bernal-Martinez L, Cuseta I and Buitrago MJ: Comparison of the Vitek 2 antifungal susceptibility system with the clinical and laboratory standards institute (CLSI) and European Committee on Antimicrobial Susceptibility Testing (EUCAST) Broth Microdilution Reference Methods and with the Sensititre YeastOne and Etest techniques for in vitro detection of antifungal resistance in yeast isolates. J Clin Microbiol 48: 1782-1786, 2010.

22. Brandt MR, Beyer CE and Stahl SM: TRPV1 Antagonists and chronic pain: Beyond thermal perception. Pharmaceuticals (Basel) 5: 114-132, 2012.

23. Nedergaard J, Bengtsson T and Cannon B: Unexpected evidence for active brown adipose tissue in adult humans. Am J Physiol Endocrinol Metab 293: E444-E452, 2007.

24. King VL, Dwoskin LP and Cassis LA: Cold exposure regulates the norepinephrine uptake transporter in rat brown adipose tissue. Am J Physiol 276: R143-R151, 1999.

25. Williamson JR, Prusiner S, Olson MS and Fukami M: Control of metabolism in brown adipose tissue. Lipids 5: 1-14, 1970.

26. Dessolin S, Schalling M, Champigny O, Lönnqvist F, Ailhaud G, Dani C and Ricquier D: Leptin gene is expressed in rat brown adipose tissue at birth. FASEB J 11: 382-387, 1997.

27. Bennett BD, Solar GP, Yuan JQ, Mathias J, Thomas GR and Matthews W: A role for leptin and its cognate receptor in hematopoiesis. Curr Biol 6: 1170-1180, 1996.

28. Sarraf P, Frederich RC, Turner EM, Ma G, Jaskowiak NT, Rivet DJ III, Flier JS, Lowell BB, Fraker DL and Alexander HR: Multiple cytokines and acute inflammation raise mouse leptin levels: Potential role in inflammatory anorexia. J Exp Med 185: 171-175, 1997.

29. Lord GM, Matarese G, Howard JK, Baker RJ, Bloom SR and Lechler RI: Leptin modulates the T-cell immune response and reverses starvation-induced immunosuppression. Nature 394: 897-901, 1998.

30. Wieland CW, Stegenga ME, Florquin S, Fantuzzi G and van der Poll T: Leptin and host defense against Gram-positive and Gram-negative pneumonia in mice. Shock 25: 414-419, 2006.

31. Kanda N and Watanabe S: Leptin enhances human beta-defensin-2 production in human keratinocytes. Endocrinology 149: 5189-5198, 2008.

32. de Jongh GJ,Zeeuwen PL, Kucharekova M,Pfundt R, van der Valk PG, Blokx W, Dogan A, Hiemstra PS, van de Kerkhof PC and Schalkwijk J: High expression levels of keratinocyte antimicrobial proteins in psoriasis compared with atopic dermatitis. J Invest Dermatol 125: 1163-1173, 2005.

33. Dhople V, Krukemeyer A and Ramamoorthy A: The human beta-defensin-3, an antibacterial peptide with multiple biological functions. Biochim Biophys Acta 1758: 1499-1512, 2006.

34. Namkung JH, Lee JE, Kim E, Kim HJ, Seo EY, Jang HY, Shin ES, Cho EY and Yang JM: Association of polymorphisms in genes encoding IL-4, IL-13 and their receptors with atopic dermatitis in a Korean population. Exp Dermatol 20: 915-919, 2011.

35. Varin A, Mukhopadhyay S, Herbein G and Gordon S: Alternative activation of macrophages by IL-4 impairs phagocytosis of pathogens but potentiates microbial-induced signalling and cytokine secretion. Blood 115: 353-362, 2010.

36. Janković BD, Popesković L, Janezić A and Lukić ML: Brown adipose tissue: Effect on immune reactions in the rat. Naturwissenschaften 61: 36, 1974.

37. Himms-Hagen J: Brown adipose tissue thermogenesis: Interdisciplinary studies. FASEB J 4: 2890-2898, 1990. 